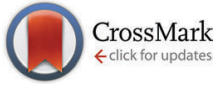

Cite this: Phys. Chem. Chem. Phys., 2014, 16, 25819

Received 6th August 2014, Accepted 7th October 2014

DOI: $10.1039 / c 4 c p 03505 d$

www.rsc.org/pccp

\section{Optical activity in the scattering of structured light}

\author{
Robert P. Cameron* and Stephen M. Barnett
}

\section{Introduction}

It is now well established in theory that a difference in response to left- and right-handed circular polarisations, or optical activity, in the scattering of light is exhibited naturally by chiral molecules ${ }^{1-5}$ and can be induced additionally in all molecules and atoms by an applied static magnetic ${ }^{5,6}$ or electric ${ }^{5,7}$ field. The phenomenon permits the extraction of information about molecules and atoms that is not readily obtainable through optical rotation or circular dichroism, owing to the subtly different physical mechanism and greater geometrical freedom involved., ${ }^{4,5}$

Many manifestations of optical activity in light scattering have now been observed in experiment. ${ }^{5,8-12}$ Natural Raman optical activity in particular has been developed into an incisive spectroscopic tool which has been employed to study large biological molecules and even intact viruses. ${ }^{5,8,11,12}$ Owing primarily to the smallness of the effects involved, there remains much to be pursued, however. Natural Rayleigh optical activity for example has thus far resisted attempts to observe it in experiment, ${ }^{5,13}$ in spite of potential applications such as the robust assignment of the absolute configurations of small chiral molecules. ${ }^{14}$

To the best of our knowledge, the theoretical and experimental approaches undertaken to date towards the phenomenon have been concerned with the illumination of molecules or atoms by single plane (or quasi plane) waves. $\dagger^{1-12,14}$ We observe, however, that optical activity in light scattering can also be probed, in general, using other types of illuminating light and that this introduces new possibilities for the study of molecules and atoms. In the present paper, we demonstrate this explicitly for natural Rayleigh optical activity which, we suggest, could be exploited as a new form of spectroscopy for chiral molecules through the use of illuminating light comprised of two plane waves that are counter propagating.

School of Physics and Astronomy, University of Glasgow, Glasgow G12 8QQ, UK.

E-mail: r.cameron.2@research.gla.ac.uk

$\dagger$ In experiment, single beams of light that resemble plane waves.
In what follows, we work within the classical domain and consider ourselves to be in an inertial frame of reference. We adopt a right-handed Cartesian coordinate system; $x, y, z$. A double appearance of an index taken from the start of the roman alphabet; $a, b, c, \ldots$, implies summation over $x, y$ and $z$. The international system of units is respected, with $\varepsilon_{0}$ and $\mu_{0}$ the electric and magnetic constants and $c=1 / \sqrt{\varepsilon_{0} \mu_{0}}$ the speed of light in vacuum. Complex quantities are indicated as such using a tilde. Unit vectors are indicated as such using a circumflex.

\section{Natural Rayleigh optical activity}

Consider a collection of $N \gg 1$ chiral molecules located at fixed positions $\mathbf{R}_{n}(n=1, \ldots, N)$. Neglecting interactions between them and assuming an absence of applied static electric and magnetic fields, we take the molecules to be randomly orientated but otherwise identical. We suppose, however, that they are illuminated by weak, monochromatic, off-resonance light of angular frequency $\omega=c k$, the length scale $2 \pi / k$ associated with which is significantly larger than each molecule. The electric field $\mathbf{E}=\mathbf{E}(\mathbf{r}, t)$ and magnetic flux density $\mathbf{B}=\mathbf{B}(\mathbf{r}, t)$ comprising the illuminating light evolve in space $\mathbf{r}$ and time $t$ as

$$
\begin{aligned}
& \mathbf{E}=\Re[\tilde{\mathbf{E}} \exp (-\mathrm{i} \omega t)], \\
& \mathbf{B}=\Re[\tilde{\mathbf{B}} \exp (-\mathrm{i} \omega t)],
\end{aligned}
$$

with the function $\Re$ yielding the real part of its argument and the complex quantities $\tilde{\mathbf{E}}=\tilde{\mathbf{E}}(\mathbf{r})$ and $\tilde{\mathbf{B}}=\tilde{\mathbf{B}}(\mathbf{r})$ satisfying

$$
\begin{gathered}
\nabla \cdot \tilde{\mathbf{E}}=0, \\
\nabla \cdot \tilde{\mathbf{B}}=0, \\
\nabla \times \tilde{\mathbf{E}}=\mathrm{i} \omega \tilde{\mathbf{B}}, \\
\nabla \times \tilde{\mathbf{B}}=-\varepsilon_{0} \mu_{0} \mathrm{i} \omega \tilde{\mathbf{E}},
\end{gathered}
$$


with $\nabla$ the gradient operator with respect to $\mathbf{r}$, as follows from the charge-free Maxwell equations: ${ }^{15}$ we regard the illuminating light as being an externally imposed influence acting upon the molecules that is (otherwise) freely propagating.

The illuminating light induces oscillations in the charge and current distributions of the molecules. In particular, the components $\mu_{a}^{(n)}=\mu_{a}^{(n)}(t), \Theta_{a b}^{(n)}=\Theta_{a b}^{(n)}(t)$ and $m_{a}^{\prime(n)}=m_{a}^{\prime(n)}(t)$ of the induced electric dipole, electric quadrupole and magnetic dipole moments of the $n$th molecule are, in a standard notation, ${ }^{5}$

$$
\begin{aligned}
\mu_{a}^{(n)} & \approx \Re\left[\tilde{\mu}_{a}^{(n)} \exp (-\mathrm{i} \omega t)\right], \\
\Theta_{a b}^{(n)} & \approx \Re\left[\tilde{\Theta}_{a b}^{(n)} \exp (-\mathrm{i} \omega t)\right], \\
m_{a}^{\prime(n)} & \approx \Re\left[\tilde{m}_{a}^{\prime(n)} \exp (-\mathrm{i} \omega t)\right],
\end{aligned}
$$

with the complex quantities $\tilde{\mu}_{a}^{(n)}, \tilde{\Theta}_{a b}^{(n)}$ and $\tilde{m}_{a}^{\prime(n)}$ related to the illuminating light as

$$
\begin{aligned}
& \tilde{\mu}_{a}^{(n)} \approx \alpha_{a b} \tilde{E}_{b}\left(\mathbf{R}_{n}\right)+\frac{1}{3} A_{a b c} \partial_{b} \tilde{E}_{c}\left(\mathbf{R}_{n}\right)-\mathrm{i} G_{a b}{ }^{\prime} \tilde{B}_{b}\left(R_{n}\right), \\
& \tilde{\Theta}_{a b}^{(n)} \approx A_{c a b} \tilde{E}_{c}\left(\mathbf{R}_{n}\right) \\
& \tilde{m}_{a^{\prime}}^{(n)} \approx \mathrm{i} G_{b a}{ }^{\prime} \tilde{E}_{b}\left(\mathbf{R}_{n}\right)
\end{aligned}
$$

for $\omega$ lying in the visible or near infrared say, with $\alpha_{a b}=\alpha_{b a}=$ $\alpha_{a b}\left(f_{\omega}\right), A_{a b c}=A_{a c b}=A_{a b c}\left(f_{\omega}\right), G_{a b}{ }^{\prime}=G_{a b}{ }^{\prime}\left(f_{\omega}\right)$ molecular polarisabilities. These oscillations generate Rayleigh scattered light in turn, ${ }^{4,5}$ the electric field $\mathbf{E}_{\mathrm{S}}=\mathbf{E}_{\mathrm{S}}(\mathbf{r}, t)$ and magnetic flux density $\mathbf{B}_{\mathrm{s}}=\mathbf{B}_{\mathrm{s}}(\mathbf{r}, t)$ of which at a position $\mathbf{R}$ of fixed magnitude $|\mathbf{R}| \gg\left|\mathbf{R}_{n}\right|$, $2 \pi / k$ are

$$
\begin{aligned}
& \mathbf{E}_{\mathrm{s}} \approx \Re\left[\tilde{\mathbf{E}}_{\mathrm{s}} \exp (-\mathrm{i} \omega t)\right], \\
& \mathbf{B}_{\mathrm{s}} \approx \Re\left[\tilde{\mathbf{B}}_{\mathrm{s}} \exp (-\mathrm{i} \omega t)\right],
\end{aligned}
$$

with the components $\tilde{E}_{\mathrm{s} a}$ and $\tilde{B}_{\mathrm{s} a}$ of the complex quantities $\tilde{\mathbf{E}}_{\mathrm{S}}=\tilde{\mathbf{E}}_{\mathrm{S}}(\hat{\mathbf{R}})$ and $\tilde{\mathbf{B}}_{\mathrm{S}}=\tilde{\mathbf{B}}_{\mathrm{S}}(\hat{\mathbf{R}})$ related to the oscillations in the charge and current distributions of the molecules as ${ }^{5}$

$$
\begin{aligned}
\tilde{E}_{\mathrm{s} a}= & \sum_{n=1}^{N} \frac{\omega^{2} \mu_{0}}{4 \pi|\mathbf{R}|} \exp \left(\mathrm{i} k\left|\mathbf{R}-\mathbf{R}_{n}\right|\right)\left\{\tilde{\mu}_{a}^{(n)}-\hat{R}_{a} \hat{R}_{b} \tilde{\mu}_{b}^{(n)}\right. \\
& \left.-\hat{R}_{b} \frac{1}{c} \varepsilon_{a b c} \tilde{m}_{c}^{\prime(n)}-\frac{\mathrm{i} k}{3} \hat{R}_{b}\left[\tilde{\Theta}_{a b}^{(n)}-\hat{R}_{a} \hat{R}_{c} \tilde{\Theta}_{b c}^{(n)}\right]\right\}, \\
\tilde{B}_{\mathrm{s} a}= & \frac{1}{c} \varepsilon_{a b c} \hat{R}_{b} \tilde{E}_{\mathrm{s} c}
\end{aligned}
$$

for $\varepsilon_{a b c}$ the rotational Levi-Civita pseudotensor defined such that $\varepsilon_{x y z}=1$. The intensity $I=I(\hat{\mathbf{R}})$ of the scattered light seen at $\mathbf{R}$ follows from Poynting's vector as

$$
I=\overline{\left\langle\left|\frac{1}{\mu_{0}} \mathbf{E}_{\mathrm{s}} \times \mathbf{B}_{\mathrm{s}}\right|\right\rangle},
$$

with an overbar indicating a cycle average and angular brackets indicating an isotropic rotational average, ${ }^{4,5}$ to account for the random orientations of the molecules. Making no assumptions beyond those described above whilst rejecting ' $A$,', ' $A G^{\prime}$ ' and ' $G^{\prime 2}$, contributions, which are anticipated to be some three orders of magnitude smaller than the smallest contributions thus retained, ${ }^{5}$ we find that we can express $I$ in terms of six properties $\mathscr{A}, \mathscr{B}, \mathscr{C}, \mathscr{D}, \mathscr{E}$ and $\mathscr{F}$ of the molecules and six related properties $W, T_{a b}, H, \mathbf{S}, N_{a b}$ and $X_{a b}$ of the illuminating light:

$$
\begin{aligned}
I \approx & \sum_{n=1}^{N} \frac{K}{|\mathbf{R}|^{2}}\left\{\mathscr{A} W\left(\mathbf{R}_{n}\right)+\mathscr{B} \hat{R}_{a} \hat{R}_{b} T_{a b}\left(\mathbf{R}_{n}\right)\right. \\
& +\omega\left[\mathscr{C} H\left(\mathbf{R}_{n}\right)+\mathscr{D} \hat{\mathbf{R}} \cdot \mathbf{S}\left(\mathbf{R}_{n}\right)\right. \\
& \left.\left.+\mathscr{E} \hat{R}_{a} \hat{R}_{b} N_{a b}\left(\mathbf{R}_{n}\right)+\mathscr{F} \hat{R}_{a} \hat{R}_{b} X_{a b}\left(\mathbf{R}_{n}\right)\right]\right\},
\end{aligned}
$$

with $K=\mu_{0}{ }^{2} c \omega^{4} / 2880 \pi^{2}$. Both $\mathscr{A}$ and $\mathscr{B}$ are equal for opposite molecular enantiomers and are thus insensitive to the chirality of the molecules, whilst $W$ and $T_{a b}$ pertain to the energy and linear momentum of the illuminating light. $\mathscr{C}, \mathscr{D}, \mathscr{E}$ and $\mathscr{F}$, however, each assume equal magnitudes but opposite signs for opposite molecular enantiomers and so are sensitive to the chirality of the molecules, whilst $H, \mathbf{S}$ and $N_{a b}$ pertain to the helicity, spin and $a b$ infra zilches of the illuminating light, these being manifestly intrinsic rotation angular momenta. ${ }^{16-21}$ Explicit expressions for these and $X_{a b}$ are given in Appendix A.

Our calculation differs from those that have been performed previously $^{1-5}$ in that the illuminating light here need not be comprised of a single plane wave of angular frequency $\omega$ but rather can be constructed from any superposition of such waves. Moreover, the molecules need not be distributed homogeneously and could instead be confined within a plane, for example. It should be noted, however, that (8) is not appropriate when the direction $\hat{\mathbf{R}}$ of observation coincides with the direction of propagation of a planewave component of the illuminating light, which will then interfere with the scattered light as Rayleigh scattering is a coherent process. $^{4,5}$ Moreover, having been derived specifically for illuminating light that is (otherwise) freely propagating in accord with the charge-free Maxwell equations, (8) is not appropriate for illuminating light the electric field of which possesses a non-vanishing irrotational component, such as may be found in the near field of a radiating structure, for example. We have refrained from exhibiting a generalised structure factor (inter-molecule cross terms ${ }^{4}$ ) in (8) as it makes no contribution in the examples that follow and in most other geometries besides.

Our general result (8) reveals in particular that natural Rayleigh optical activity, as manifest in $I$, can be utilised to extract information about the chirality of the molecules using essentially any type of illuminating light possessing nonvanishing helicity, spin and/or $a b$ infra zilches. ${ }^{16-21}$ A single circularly polarised plane wave is, perhaps, the most obvious example of such light and is examined in Section 3. It is not the only one, however: ${ }^{22}$ as we will demonstrate in Sections 4-6, types of illuminating light comprised of two plane waves that are counter propagating can carry these angular momenta in novel ways and thus enable new possibilities. In identifying these, we were guided by symmetry considerations. In particular, it is necessary for the illuminating light together with the direction of observation to be of chiral character in order that $I$ itself be capable of distinguishing between opposite molecular enantiomers, as is inherent, of course, in (8). 


\section{Example zero: circularly polarised illuminating light}

As a check on the validity of (8) and for comparison in what follows, let us begin now by following previous approaches ${ }^{1-5}$ and considering illuminating light comprised of a single circularly polarised plane wave of amplitude $E_{0}$ propagating in the $z$ direction as

$$
\begin{gathered}
\tilde{\mathbf{E}}_{ \pm}^{(0)}=E_{0}(\hat{\mathbf{x}} \pm \mathrm{i} \hat{\mathbf{y}}) \exp (\mathrm{i} k z), \\
\tilde{\mathbf{B}}_{ \pm}^{(0)}=E_{0}(\mp \mathrm{i} \hat{\mathbf{x}}+\hat{\mathbf{y}}) \exp (\mathrm{i} k z) / c,
\end{gathered}
$$

with $\hat{\mathbf{x}}, \hat{\mathbf{y}}$ (and $\hat{\mathbf{z}}$ ) unit vectors in the $x, y$ (and $z$ ) directions and where the upper and lower signs yield left- and right-handed circular polarisation in the optics convention, ${ }^{15}$ which we adopt. This is both the prototypical type of light possessing non-vanishing helicity, spin and $a b$ infra zilches ${ }^{17,18}$ and the prototypical type of chiral light: ${ }^{5}$ as time passes, $\mathbf{E}$ and $\mathbf{B}$ rotate with a phase that varies in $z$ such that they trace out cylindrical helices, the chiralities of which differ for the upper and lower signs in (9). Accordingly

$$
\begin{gathered}
W_{ \pm}^{(0)}=\varepsilon_{0} E_{0}{ }^{2}, \\
\hat{R}_{a} \hat{R}_{b} T_{a b \pm}^{(0)}=\hat{R}_{z}{ }^{2} \varepsilon_{0} E_{0}{ }^{2}, \\
H_{ \pm}^{(0)}= \pm \varepsilon_{0} E_{0}{ }^{2} / \omega, \\
\hat{\mathbf{R}} \cdot \mathbf{S}_{ \pm}^{(0)}= \pm \hat{R}_{z} \varepsilon_{0} E_{0}{ }^{2} / \omega, \\
\hat{R}_{a} \hat{R}_{b} N_{a b \pm}^{(0)}= \pm \hat{R}_{z}{ }^{2} \varepsilon_{0} E_{0}{ }^{2} / \omega, \\
\hat{R}_{a} \hat{R}_{b} X_{a b \pm}^{(0)}=0,
\end{gathered}
$$

where the upper and lower signs correspond to those in (9). Taking the molecules to be homogeneously distributed around the origin, we find then that

$$
I_{ \pm}^{(0)} \approx \frac{\varepsilon_{0} K N E_{0}^{2}}{|\mathbf{R}|^{2}}\left[\mathscr{A}+\mathscr{B} \hat{R}_{z}^{2} \pm\left(\mathscr{C}+\mathscr{D} \hat{R}_{z}+\mathscr{E} \hat{R}_{z}^{2}\right)\right]
$$

where the upper and lower signs again correspond to those in (9). Natural Rayleigh optical activity, as manifest in $I_{ \pm}^{(0)}$, is thus attributable to the non-vanishing helicity, spin and $a b$ infra zilches possessed by the illuminating light: $I_{ \pm}^{(0)}$ differs for leftand right-handed circular polarisations because $H_{ \pm}^{(0)}, \mathbf{S}_{ \pm}^{(0)}$ and $N_{a b \pm}^{(0)}$ do. For right-angled observation in particular, with $\hat{\mathbf{R}}=\hat{\mathbf{x}}$ say;

$$
I_{ \pm}^{(0)}(\hat{\mathbf{x}}) \approx \frac{\varepsilon_{0} K N E_{0}^{2}}{|\mathbf{R}|^{2}}(\mathscr{A} \pm \mathscr{C}) .
$$

This situation is depicted in Fig. 1.

This phenomenon is neatly quantified by the circular intensity difference $\Delta^{(0)}=\Delta^{(0)}(\hat{\mathbf{R}})$ defined as $\ddagger^{2}$

$$
\Delta^{(0)}=\frac{I_{+}^{(0)}-I_{-}^{(0)}}{I_{+}^{(0)}+I_{-}^{(0)}},
$$

$\$ \Delta^{(0)}$ differs in sign from the circular intensity difference introduced in ref. 2 . which has equal magnitudes but opposite signs for opposite molecular enantiomers. Without loss of generality, we take $\hat{\mathbf{R}}=\sin \phi \hat{\mathbf{x}}+\cos \phi \hat{\mathbf{z}}$ and find that

$$
\Delta^{(0)}(\sin \phi \hat{\mathbf{x}}+\cos \phi \hat{\mathbf{z}}) \approx \frac{\mathscr{C}+\mathscr{D} \cos \phi+\mathscr{E} \cos ^{2} \phi}{\mathscr{A}+\mathscr{B} \cos ^{2} \phi},
$$

which is the anticipated result. ${ }^{3}$ For right-angled observation in particular, ${ }^{2,4,5}$ with $\hat{\mathbf{R}}=\hat{\mathbf{x}}$;

$$
\Delta^{(0)}(\hat{\mathbf{x}}) \approx \frac{\mathscr{C}}{\mathscr{A}}=\frac{2\left(45 a G^{\prime}-13 \beta_{G}^{2}+\beta_{A}^{2}\right)}{c\left(45 a^{2}+13 \beta^{2}\right)} .
$$

Owing primarily to the contribution made in the denominator by $a^{2}, \Delta^{(0)}$ is rather small and, to the best of our knowledge, has not yet been observed in experiment for chiral molecules: $:^{5,13}$ calculated magnitudes of $\Delta^{(0)}$ typically lie between $10^{-6}$ and $10^{-4} \cdot{ }^{14}$ We note, however, that experimental results have been reported for large biological structures. ${ }^{23}$

\section{Example one: superchiral illuminating light}

Following a procedure recently suggested ${ }^{24}$ and demonstrated $^{25}$ for luminescence-detected circular dichroism, we observe here the possibility of using so-called superchiral illuminating light, rather than illuminating light comprised of a single circularly polarised plane wave, to ensure that a larger fraction of $I$ is sensitive to the chirality of the molecules, albeit at the expense of an overall reduction in $I$. We associate with this illuminating light, a quantity analogous to $\Delta^{(0)}(\hat{\mathbf{x}})$ that can be made larger in magnitude and may, therefore, be more amenable to observation in experiment.

Consider then a superposition of two circularly polarised plane waves of opposite handedness, the first of which has amplitude $E_{1} / \sqrt{2}$ and propagates in the $z$ direction whilst the second has amplitude $E_{2} / \sqrt{2} \neq E_{1} / \sqrt{2}$ and propagates in the $-z$ direction as

$$
\begin{aligned}
& \tilde{\mathbf{E}}_{ \pm}^{(1)}=E_{1}(\hat{\mathbf{x}} \pm \mathrm{i} \hat{\mathbf{y}}) \exp (\mathrm{i} k z) / \sqrt{2}-E_{2}(\hat{\mathbf{x}} \pm \mathrm{i} \hat{\mathbf{y}}) \exp (-\mathrm{i} k z) / \sqrt{2} \\
& \tilde{\mathbf{B}}_{ \pm}^{(1)}=E_{1}(\mp \mathrm{i} \hat{\mathbf{x}}+\hat{\mathbf{y}}) \exp (\mathrm{i} k z) / \sqrt{2} c-E_{2}(\mp \mathrm{i} \hat{\mathbf{x}}-\hat{\mathbf{y}}) \exp (-\mathrm{i} k z) / \sqrt{2} c
\end{aligned}
$$

where the upper and lower signs distinguish the cases in which the first wave is left- or right-handed. This illuminating light is manifestly chiral as, at any given $t, \mathbf{E}$ and $\mathbf{B}$ twist helically in $z$, the sense of twist depending on $\operatorname{sgn}\left(E_{1}-E_{2}\right)$ whilst differing for the upper and lower signs in (16). As time passes, these helical patterns themselves rotate rigidly about the $z$ axis, with the sense of rotation differing for the upper and lower signs in (16). In the vicinity of the $z=0$ plane, $\mathbf{E}$ twists unusually fast in $z$, doing so at the cost of a reduced magnitude: a superoscillatory phenomenon. ${ }^{26}$ In contrast, $\mathbf{B}$ twists rather slowly in the vicinity of $z=0$, but is of relatively large magnitude. The effect becomes more pronounced as $\left|E_{1}-E_{2}\right| \rightarrow 0$. 

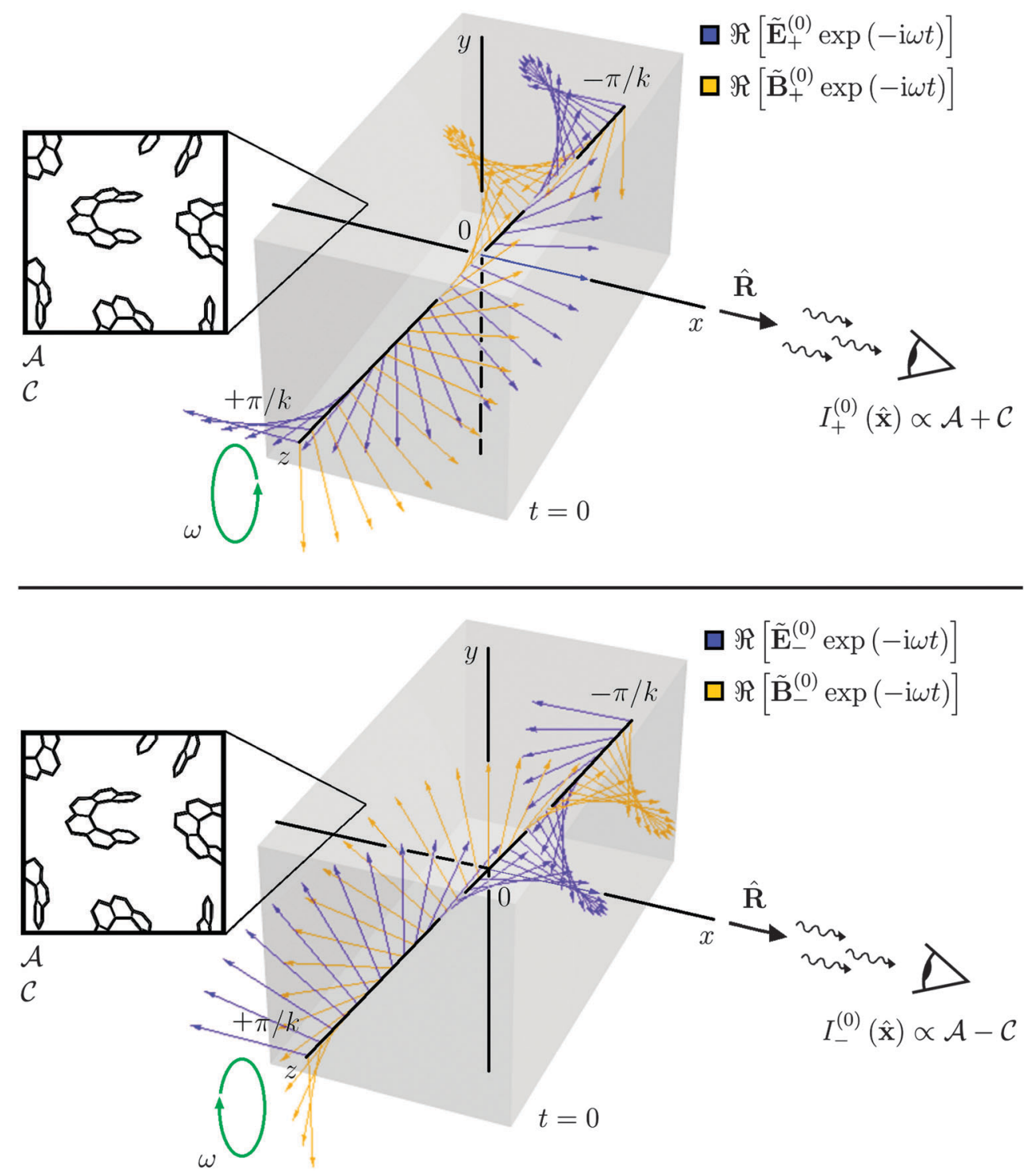

Fig. 1 Illuminating light comprised of a single circularly polarised plane wave is scattered differently by the molecules depending upon whether it is leftor right-handed.

Let us suppose then that the molecules are distributed homogeneously in the $z=0$ plane around the origin. Adopting a right-angled observation geometry with $\hat{\mathbf{R}}=\hat{\mathbf{x}}$, we find that

$$
I_{ \pm}^{(1)}(\hat{\mathbf{x}}) \approx \frac{\varepsilon_{0} K N\left(E_{1}-E_{2}\right)}{2|\mathbf{R}|^{2}}\left[\mathscr{A}\left(E_{1}-E_{2}\right) \pm \mathscr{C}\left(E_{1}+E_{2}\right)\right],
$$

where the upper and lower signs correspond to those in (16). Comparing (17) with (12), we see that the chirally insensitive $\mathscr{A}$ contribution to $I_{ \pm}^{(1)}(\hat{\mathbf{x}})$ is reduced relative to the chirally sensitive $\mathscr{C}$ contribution, albeit at the expense of an overall reduction in $I_{ \pm}^{(1)}(\hat{\mathbf{x}})$. This occurs because the chirally insensitive $\mathscr{A}$ contribution to $I_{ \pm}^{(1)}(\hat{\mathbf{x}})$ is driven by $\mathbf{E}$ alone through $\bar{W}$ whilst the chirally sensitive $\mathscr{C}$ contribution is driven instead by both $\mathbf{E}$ and $\mathbf{B}$ through $\bar{H}$ : in the latter case, the unusually high degree of twisting exhibited by $\mathbf{E}$ in the vicinity of the $z=0$ plane, where the molecules reside, together with the relatively large magnitude of B compensates somewhat for the small magnitude of $\mathbf{E}$ there. The situation is depicted in Fig. 2.

We quantify this phenomenon through a generalised intensity difference $\chi^{(1)}$ defined as

$$
\begin{aligned}
\chi^{(1)}= & \frac{I_{+}^{(1)}(\hat{\mathbf{x}})-I_{-}^{(1)}(\hat{\mathbf{x}})}{I_{+}^{(1)}(\hat{\mathbf{x}})+I_{-}^{(1)}(\hat{\mathbf{x}})} \\
& \approx \frac{E_{1}+E_{2}}{E_{1}-E_{2}} \frac{\mathscr{A}}{\mathscr{A}} \\
& \approx \frac{E_{1}+E_{2}}{E_{1}-E_{2}} \Delta^{(0)}(\hat{\mathbf{x}}),
\end{aligned}
$$

which has equal magnitudes but opposite signs for opposite molecular enantiomers. This $\chi^{(1)}$ should be equal to or greater in magnitude than $\Delta^{(0)}(\hat{\mathbf{x}})$ and diverges, in fact, as $\left|E_{1}-E_{2}\right| \rightarrow 0$ (and $I_{ \pm}^{(1)}(\hat{\mathbf{x}}) \rightarrow 0$ ). In reality, such enhancements of $\chi^{(1)}$ relative 

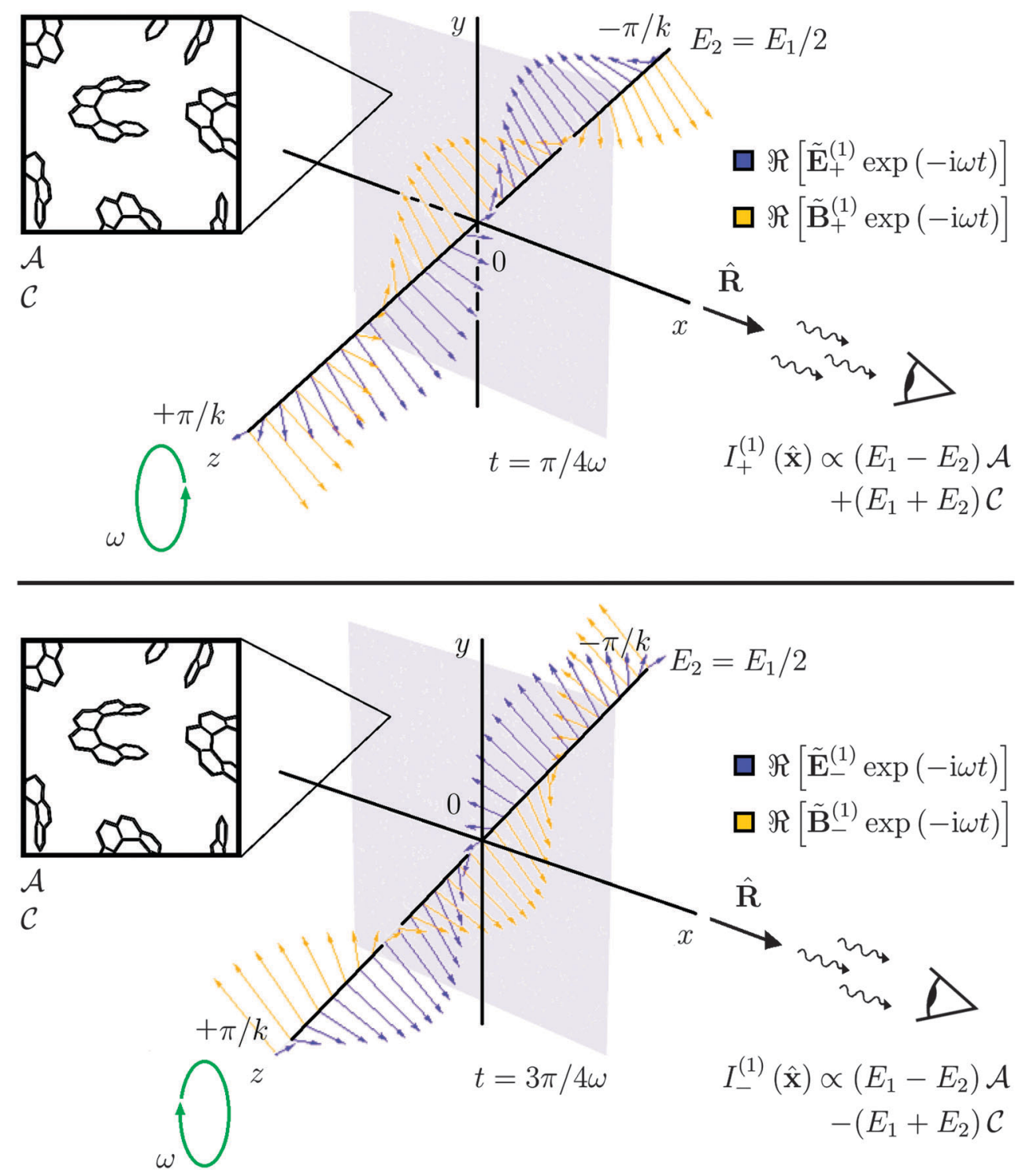

Fig. 2 Superchiral illuminating light can be employed to ensure that a larger fraction of the intensity of the scattered light is sensitive to the chirality of the molecules, as compared to illuminating light comprised of a single circularly polarised plane wave.

to $\Delta^{(0)}$ are limited by contributions to the $m_{a}^{\prime(n)}$ induced by $\mathbf{B}$, which we have omitted explicitly from our analysis. Nevertheless, gains up to three orders of magnitude may be possible. ${ }^{4,5,24,25}$

\section{Example two: $\sigma-\sigma$ illuminating light}

We observe now the possibility of using so-called $\sigma-\sigma$ illuminating light, rather than illuminating light comprised of a single circularly polarised plane wave, to remove isotropic contributions to $I$ whilst retaining both chirally insensitive and chirally sensitive anisotropic contributions. We associate with this illuminating light, a quantity analogous to $\Delta^{(0)}(\hat{\mathbf{x}})$ that is significantly larger in magnitude whilst offering different and perhaps more desirable information about the chirality of the molecules. This quantity may, therefore, be more suitable for observation in experiment. $\sigma-\sigma$ light is employed for example in the laser cooling of atoms. ${ }^{27}$

Consider then a superposition of two circularly polarised plane waves of the same handedness and equal amplitude $E_{0} / \sqrt{2}$, the first of which propagates in the $z$ direction whilst the second propagates in the $-z$ direction as

$$
\begin{aligned}
& \tilde{\mathbf{E}}_{ \pm}^{(2)}=E_{0}(\hat{\mathbf{x}} \pm \mathrm{i} \hat{\mathbf{y}}) \exp (\mathrm{i} k z) / \sqrt{2}+E_{0}(\hat{\mathbf{x}} \mp \mathrm{i} \hat{\mathbf{y}}) \exp (-\mathrm{i} k z) / \sqrt{2} \\
& \tilde{\mathbf{B}}_{ \pm}^{(2)}=E_{0}(\mp \mathrm{i} \hat{\mathbf{x}}+\hat{\mathbf{y}}) \exp (\mathrm{i} k z) / \sqrt{2} c+E_{0}(\mp \mathrm{i} \hat{\mathbf{x}}-\hat{\mathbf{y}}) \exp (-\mathrm{i} k z) / \sqrt{2} c
\end{aligned}
$$

where the upper and lower signs distinguish the cases in which the waves are left- or right-handed. This illuminating light is manifestly chiral, as $\mathbf{E}$ and $\mathbf{B}$ oscillate linearly and parallel to each other at each $z$ whilst the plane in which they oscillate 
twists helically in $z$, the sense of twist differing for the upper and lower signs in (19) whilst $\mathbf{E}$ lags or leads $\mathbf{B}$ by a quarter cycle. In the $z=0$ plane, $\mathbf{E}$ and $\mathbf{B}$ lie parallel to the $x$ axis.

Let us suppose then that the molecules are distributed homogeneously in the $z=0$ plane around the origin. Adopting a right-angled observation geometry with $\hat{\mathbf{R}}=\hat{\mathbf{x}}$, we find that

$$
I_{ \pm}^{(2)}(\hat{\mathbf{x}}) \approx \frac{\varepsilon_{0} K N E_{0}^{2}}{|\mathbf{R}|^{2}}[(\mathscr{A}-\mathscr{B}) \pm(\mathscr{C}-\mathscr{E})]
$$

where the upper and lower signs correspond to those in (19). Evidently, $I_{ \pm}^{(2)}(\hat{\mathbf{x}})$ contains no isotropic contributions, either chirally insensitive: $a^{2}$, or chirally sensitive: $a G^{\prime}$. It does, however, possess non-vanishing anisotropic contributions, both chirally insensitive: $\beta^{2}$, and chirally sensitive: $\beta_{G}{ }^{2}$ and $\beta_{A}{ }^{2}$. This may be understood simply by recalling that an oscillating electric dipole moment radiates no energy on axis and so an isotropically polarisable molecular species would, to the order of present interest, exhibit no scattering in the direction $\hat{\mathbf{R}}=\hat{\mathbf{x}}$ of observation as the latter lies parallel to the (electric-dipole- inducing) $\mathbf{E}$ vectors in the $z=0$ plane where the molecules reside. The situation is depicted in Fig. 3.

We quantify this phenomenon through a generalised intensity difference $\Lambda^{(2)}$ defined as

$$
\begin{aligned}
\Lambda^{(2)}= & \frac{I_{+}^{(2)}(\hat{\mathbf{x}})-I_{-}^{(2)}(\hat{\mathbf{x}})}{I_{+}^{(2)}(\hat{\mathbf{x}})+I_{-}^{(2)}(\hat{\mathbf{x}})} \\
& \approx \frac{\mathscr{C}-\mathscr{E}}{\mathscr{A}-\mathscr{B}} \\
& =\frac{2\left(\beta_{A}^{2}-3 \beta_{G}^{2}\right)}{3 c \beta^{2}}
\end{aligned}
$$

which has equal magnitudes but opposite signs for opposite molecular enantiomers. This $\Lambda^{(2)}$ should be larger than $\Delta^{(0)}(\hat{\mathbf{x}})$ by around two orders of magnitude ${ }^{14}$ owing to the absence of a contribution from $a^{2}$ in the denominator. Moreover, $\Lambda^{(2)}(\hat{\mathbf{x}})$ offers different and perhaps more desirable information about the chirality of the molecules than $\Delta^{(0)}(\hat{\mathbf{x}})$ as its numerator is comprised solely of the quantities $\beta_{G}{ }^{2}$ and $\beta_{A}{ }^{2}$ of particular interest. We note that $\Lambda^{(2)}$ is -1 times the familiar depolarised
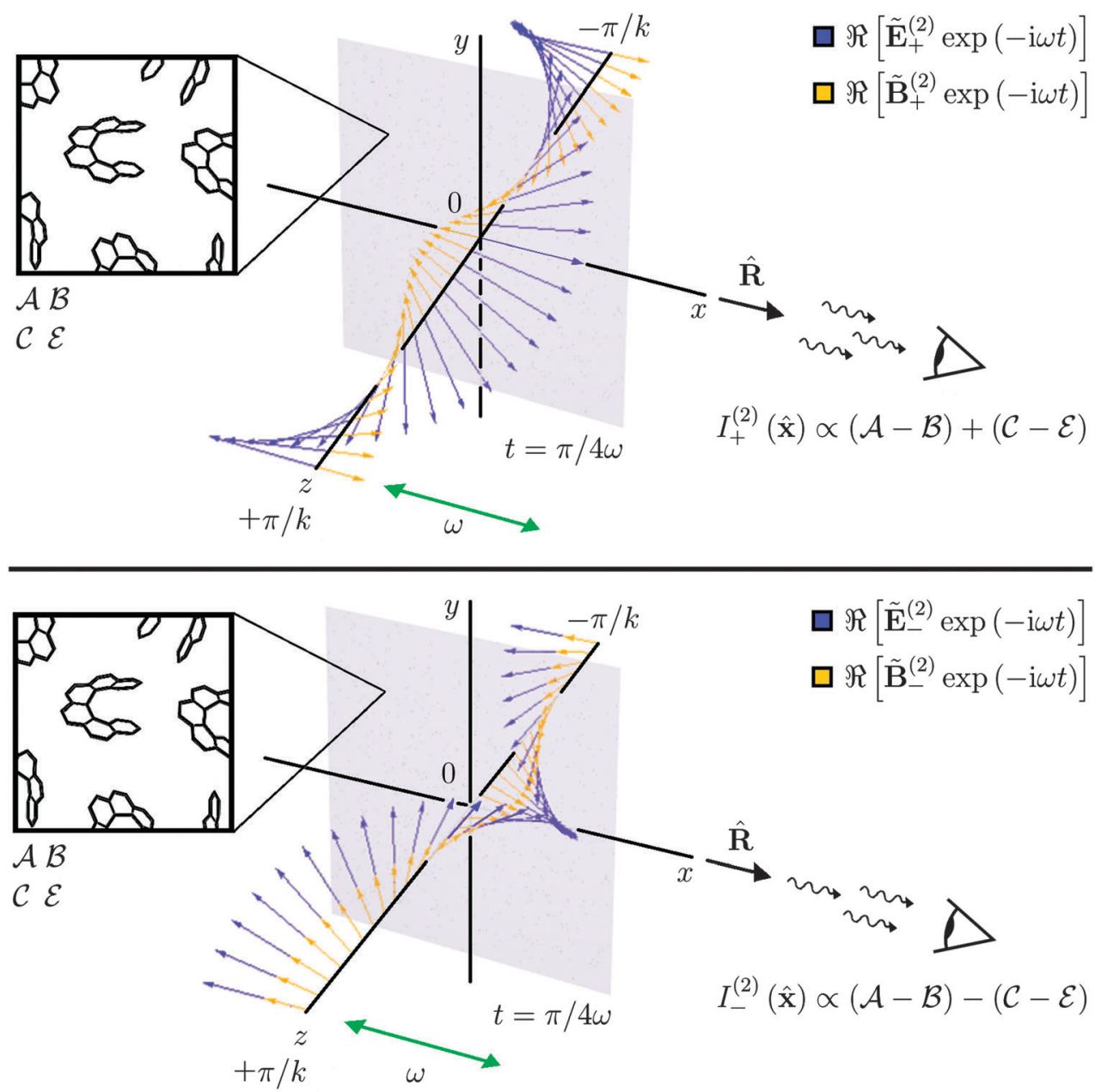

Fig. $3 \sigma-\sigma$ illuminating light can be employed to ensure that the intensity of the scattered light contains no isotropic contributions whilst still being sensitive to the chirality of the molecules. 
right-angled circular intensity difference. ${ }^{4,5}$ The latter, however, requires analysed measurements of the intensities of scattered light polarised perpendicular to the scattering plane and is prone to spurious effects, ${ }^{13}$ owing to the relatively large intensities of scattered light polarised parallel to the scattering plane. In contrast, $\Lambda^{(2)}$ requires measurement only of unanalysed scattered intensities and should, therefore, be robust in this regard. Theoretical predictions of the variation of the

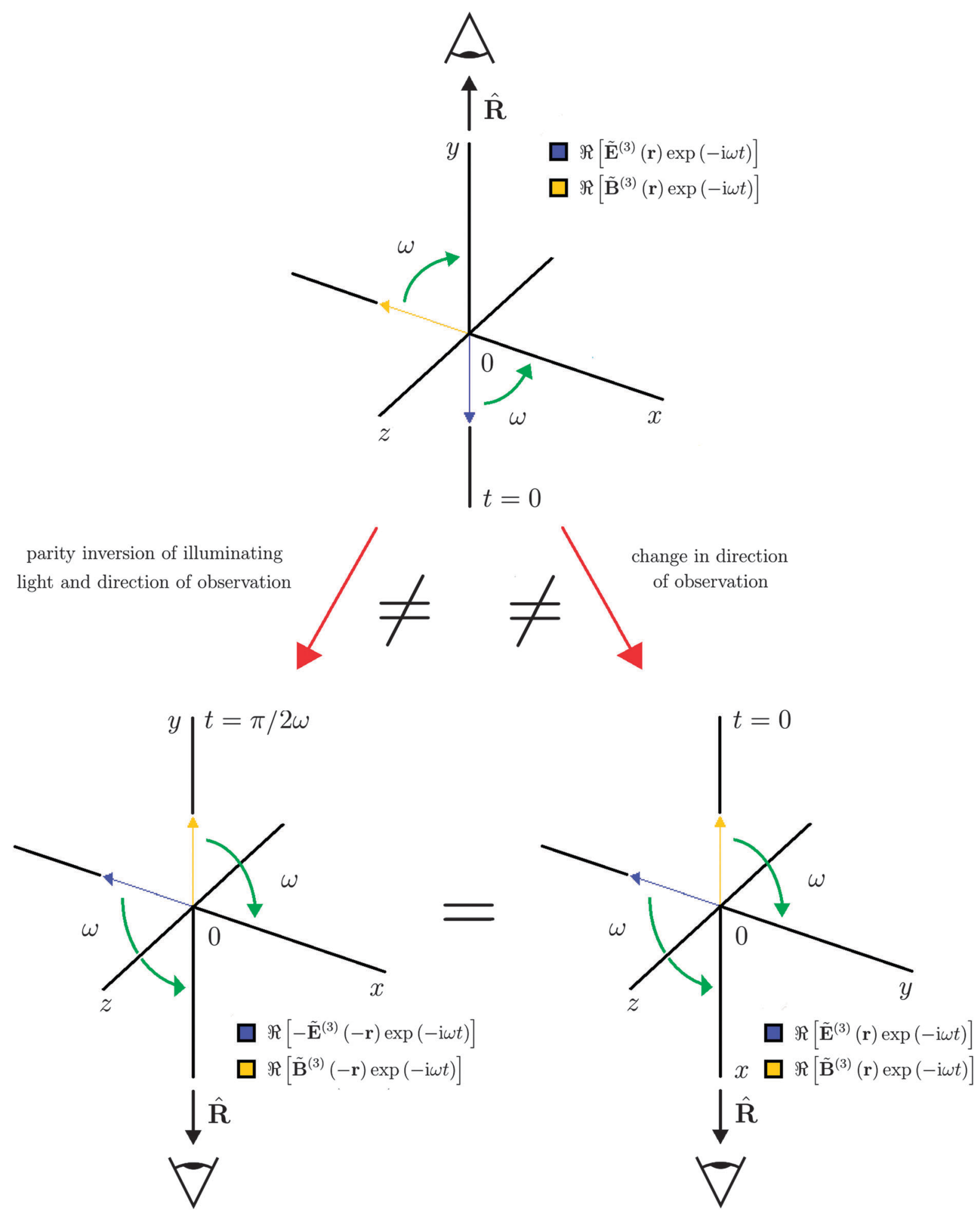

Fig. 4 A parity inversion of the lin $\perp$ lin illuminating light and the direction of observation $\hat{\mathbf{R}}=\hat{\mathbf{y}}$ through the origin can be mimicked in the $z=0$ plane by leaving the light unaltered and changing the direction of observation from $\hat{\mathbf{R}}=\hat{\mathbf{y}}$ to $\hat{\mathbf{R}}=\hat{\mathbf{x}}$. 
familiar depolarised right-angled circular intensity difference (and hence, $\Lambda^{(2)}$ ) with frequency for various molecules can be seen in the work of Züber, Wipf and Beratan. ${ }^{14}$

\section{Example three: lin $\perp$ lin illuminating light}

We observe finally the novel possibility of using so-called lin $\perp$ lin illuminating light which is essentially achiral, rather than illuminating light comprised of a single circularly polarised plane wave, to extract information about the chirality of the molecules through $I$. We associate with this illuminating light a quantity analogous to
$\Delta^{(0)}(\hat{\mathbf{x}})$ that is impervious to spurious contributions attributable to circular dichroism whilst being of a different form. Lin $\perp$ lin light is employed for example in the laser cooling of atoms. ${ }^{27}$

Consider then a superposition of two linearly polarised plane waves of equal amplitude $E_{0}$, the first of which is polarised along the $x$ axis and propagates in the $z$ direction whilst the second is polarised along the $y$ axis and propagates in the $-z$ direction as

$$
\begin{gathered}
\tilde{\mathbf{E}}^{(3)}=E_{0} \mathrm{i} \hat{\mathbf{x}} \exp (\mathrm{i} k z)-E_{0} \hat{\mathbf{y}} \exp (-\mathrm{i} k z), \\
\tilde{\mathbf{B}}^{(3)}=E_{0} \mathrm{i} \hat{\mathbf{y}} \exp (\mathrm{i} k z) / c-E_{0} \hat{\mathbf{x}} \exp (-\mathrm{i} k z) / c .
\end{gathered}
$$

It should be noted that we only have one form of illuminating light here, in contrast to examples zero, one and two where
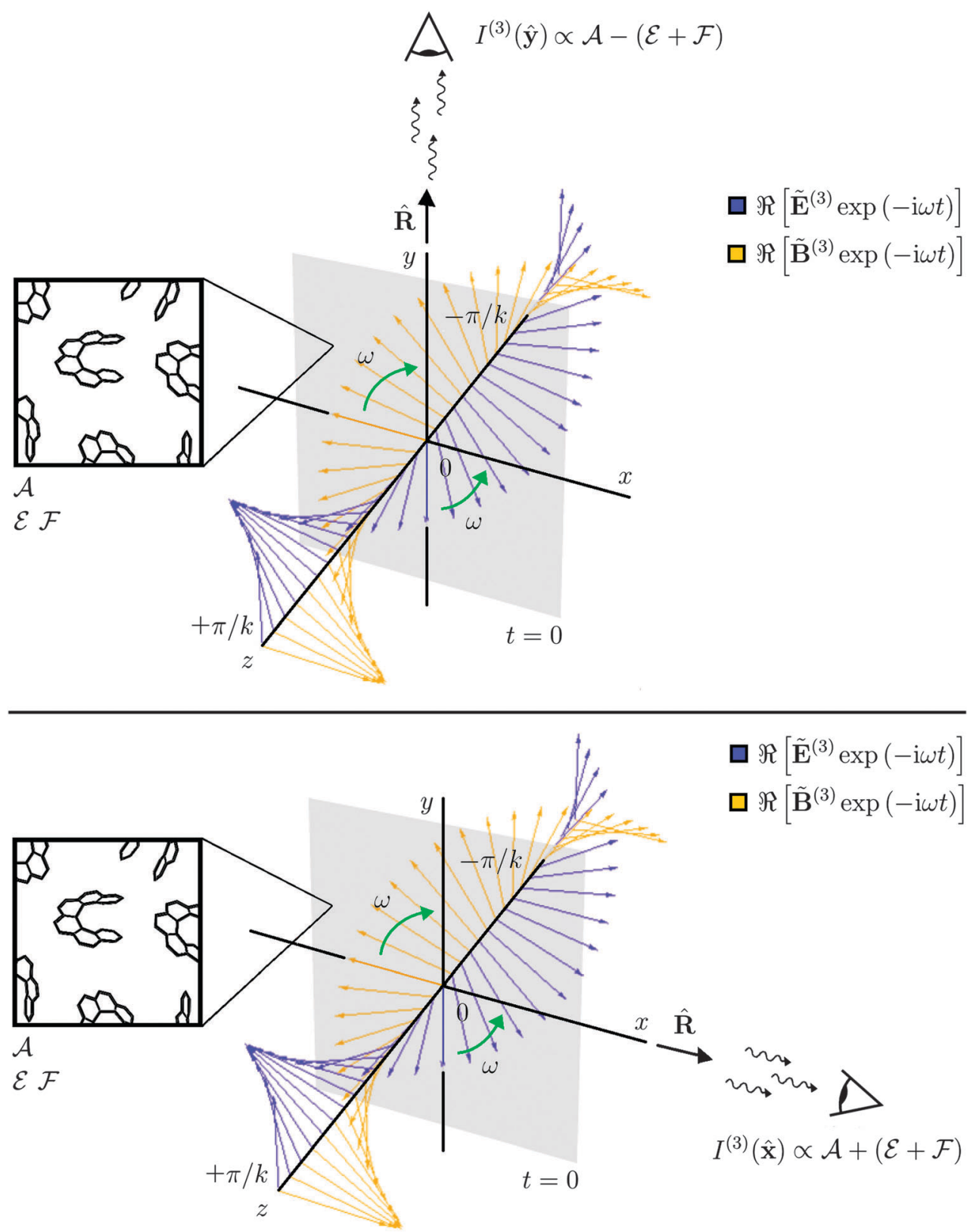

Fig. 5 Lin $\perp$ lin illuminating light, which is by itself essentially achiral, can be employed to probe the chirality of the molecules by making explicit use of the degree of freedom that is the direction in which the intensity of scattered light is observed. 
there were two forms of illuminating light which we distinguished using plus and minus signs. In the $z=0$ plane, $\mathbf{E}$ and $\mathbf{B}$ rotate in opposite directions which is, by itself, an essentially achiral configuration. The combination of $\mathbf{E}, \mathbf{B}$ and the direction of observation $\hat{\mathbf{R}}$, however, is chiral in general. In particular, a parity inversion of the illuminating light and the direction of observation $\hat{\mathbf{R}}=\hat{\mathbf{y}}$ through the origin yields a new configuration not superposable upon the old. An essentially equivalent transformation is invoked, however, by leaving the illuminating light unaltered and changing the direction of observation from $\hat{\mathbf{R}}=\hat{\mathbf{y}}$ to $\hat{\mathbf{R}}=\hat{\mathbf{x}}$. This is depicted in Fig. 4 .

Let us suppose then that the molecules are distributed homogeneously in the $z=0$ plane around the origin. We then find that

$$
\begin{aligned}
& I^{(3)}(\hat{\mathbf{x}}) \approx \frac{\varepsilon_{0} N K E_{0}^{2}}{|\mathbf{R}|^{2}}[\mathscr{A}+(\mathscr{E}+\mathscr{F})], \\
& I^{(3)}(\hat{\mathbf{y}}) \approx \frac{\varepsilon_{0} N K E_{0}^{2}}{|\mathbf{R}|^{2}}[\mathscr{A}-(\mathscr{E}+\mathscr{F})] .
\end{aligned}
$$

Evidently, information about the chirality of the molecules can be extracted simply by contrasting $I^{(3)}(\hat{\mathbf{x}})$ and $I^{(3)}(\hat{\mathbf{y}})$. This is of course possible owing to the equivalence described above. The situation is depicted in Fig. 5.

We quantify this phenomenon through a generalised intensity difference $\Upsilon^{(3)}$ defined as

$$
\begin{aligned}
\Upsilon^{(3)} & =\frac{I^{(3)}(\hat{\mathbf{y}})-I^{(3)}(\hat{\mathbf{x}})}{I^{(3)}(\hat{\mathbf{y}})+I^{(3)}(\hat{\mathbf{x}})} \\
& \approx-\frac{\mathscr{E}+\mathscr{F}}{\mathscr{A}} \\
& =\frac{2\left(45 a G^{\prime}+\beta_{G}{ }^{2}-\beta_{A}{ }^{2}\right)}{c\left(45 a^{2}+13 \beta^{2}\right)},
\end{aligned}
$$

which has equal magnitudes but opposite signs for opposite molecular enantiomers. This $Y^{(3)}$ is of a different character, of course, to $\Delta^{(0)}, \chi^{(1)}$ and $\Lambda^{(2)}$ as it is dependent upon scattered intensities associated with one form of illuminating light rather than two. It should be comparable in magnitude to $\Delta^{(0)}(\hat{\mathbf{x}})^{14}$ but offers somewhat different information about the chirality of the molecules: the contributions made by $\beta_{G}{ }^{2}$ and $\beta_{A}{ }^{2}$ to $\Upsilon^{(3)}$ are of opposite sign to those in $\Delta^{(0)}(\hat{\mathbf{x}})$ and the former is 13 times smaller. Although we have assumed the illuminating light to be off resonance, there will always exist in reality some absorption of the illuminating light by the molecules. Owing to circular dichroism: the differential absorption of left- and right-handed circularly polarised light, $\Delta^{(0)}(\hat{\mathbf{x}}), \chi^{(1)}$ and $\Lambda^{(2)}$ will therefore suffer from spurious contributions attributable not to light scattering but rather, to luminescence. By its very nature, $\Upsilon^{(3)}$, however, is impervious to such contributions. Moreover, lin $\perp$ lin illuminating light will be absorbed at the same rate by opposite molecular enantiomers, as it is essentially achiral.

\section{Discussion}

We have demonstrated that natural Rayleigh optical activity can be probed using illuminating light comprised of two plane waves that are counter propagating and that this permits new possibilities for the study of molecular chirality. Exploitation of these possibilities requires, however, that the scattering molecules be confined to a plane, in which case their number and hence, the scattered intensity, is necessarily reduced relative to that attainable in a fully homogeneous sample. It is unclear to us at present whether this limitation can be overcome simply. In experiment, such confinement might be realised by depositing the molecules onto a surface. ${ }^{30}$ of course, additional effects associated with the surface, such as reflection and refraction of the illuminating light and molecular orientation, ${ }^{31}$ would then have to be considered with care.

For our two-plane-wave examples, we restricted our attention to right-angled observation which is particularly well suited to experiment as it 'avoids' the illuminating light as much as possible. Nevertheless, more information about the chirality of the molecules may be extracted by exploring other scattering geometries, as has been suggested for illuminating light comprised of single circularly polarised plane waves. ${ }^{3}$ Our approach has been centred upon the unanalysed scattered intensity as this is, perhaps, the most readily measurable property of the scattered light. The polarisation properties of the scattered light remain to be explored, however, and may yield additional possibilities. Finally, we highlight the fact that analogous approaches to those undertaken in the present paper can be pursued for other manifestations of optical activity in light scattering. We shall return to these tasks elsewhere.

\section{Appendix A: definitions}

$$
\begin{gathered}
\mathscr{A}, \mathscr{B}, \mathscr{C}, \mathscr{D} \mathscr{E} \text { and } \mathscr{F} \text { pertain to the molecules as } \\
\mathscr{A}=2\left(45 a^{2}+13 \beta^{2}\right), \\
\mathscr{B}=2\left(45 a^{2}+\beta^{2}\right), \\
\mathscr{C}=4\left(45 a G^{\prime}-13{\beta_{G}}^{2}+{\beta_{A}}^{2}\right) / c, \\
\mathscr{D}=8\left(-45 a G^{\prime}+5{\beta_{G}}^{2}+3{\beta_{A}}^{2}\right) / c, \\
\mathscr{E}=4\left(-45 a G^{\prime}-\beta_{G}{ }^{2}-3 \beta_{A}{ }^{2}\right) / c, \\
\mathscr{F}=16 \beta_{A}{ }^{2} / c,
\end{gathered}
$$

for

$$
\begin{gathered}
a^{2}=\alpha_{a a} \alpha_{b b} / 9, \\
\beta^{2}=\left(3 \alpha_{a b} \alpha_{a b}-\alpha_{a a} \alpha_{b b}\right) / 2, \\
a G^{\prime}=\alpha_{a a} G_{b b}{ }^{\prime} / 9, \\
\beta_{G}^{2}=\left(3 \alpha_{a b} G_{a b}{ }^{\prime}-\alpha_{a a} G_{b b}{ }^{\prime}\right) / 2, \\
\beta_{A}{ }^{2}=\omega \varepsilon_{a b c} \alpha_{a d} A_{b c d} / 2 .
\end{gathered}
$$

$a^{2}$ and $\beta^{2}$ do not distinguish between opposite molecular enantiomers and are strictly positive. They can be measured 
through a combination of optical refraction and depolarised Rayleigh scattering experiments. ${ }^{3-5} \beta^{2}$ is typically an order of magnitude smaller than $a^{214}$ and vanishes entirely for an isotropic molecule. ${ }^{4,5} a G^{\prime}, \beta_{G}{ }^{2}$ and $\beta_{A}{ }^{2}$ do distinguish between opposite molecular enantiomers however, by taking on equal magnitudes but opposite signs. $§ a G^{\prime}$ can be measured through a combination of optical refraction and optical rotation experiments. ${ }^{3-5}$ In contrast, $\beta_{G}{ }^{2}$ and $\beta_{A}{ }^{2}$ cannot be readily measured by other means and they are, therefore, quantities of particular interest. ${ }^{5,14} a G^{\prime} / c, \beta_{G}{ }^{2} / c$ and $\beta_{A}{ }^{2} / c$ are typically three to five orders of magnitude smaller than $a^{2}{ }^{4,5}$

$W=W(\mathbf{r}), T_{a b}=T_{a b}(\mathbf{r}), H=H(\mathbf{r}), \mathbf{S}=\mathbf{S}(\mathbf{r}), N_{a b}=N_{a b}(\mathbf{r})$ and $X_{a b}=$ $X_{a b}(\mathbf{r})$ pertain to the illuminating light as

$$
\begin{aligned}
& W=2 \times \overline{\varepsilon_{0} \mathbf{E} \cdot \mathbf{E} / 2}, \\
& T_{a b}=2 \times \overline{\varepsilon_{0}\left(\delta_{a b} \mathbf{E} \cdot \mathbf{E}-2 E_{a} E_{b}\right) / 2}, \\
& H=\overline{\varepsilon_{0} c\left(\mathbf{A}^{\perp} \cdot \mathbf{B}-\mathbf{C}^{\perp} \cdot \mathbf{E}\right) / 2}, \\
& \mathbf{S}=2 \times \overline{\varepsilon_{0} \mathbf{E} \times \mathbf{A}^{\perp} / 2}, \\
& N_{a b}=\overline{\varepsilon_{0} c\left[\delta_{a b}\left(\mathbf{A}^{\perp} \cdot \mathbf{B}-\mathbf{C}^{\perp} \cdot \mathbf{E}\right)-A_{a}^{\perp} B_{b}-A_{b}^{\perp} B_{a}+C_{a}^{\perp} E_{b}+C_{b}^{\perp} E_{a}\right] / 2}, \\
& X_{a b}=\overline{\varepsilon_{0} c\left[\partial_{d}\left(\varepsilon_{b c d} A_{b}^{\perp} A_{c}^{\perp}+\varepsilon_{b c d} A_{a}^{\perp} A_{c}^{\perp}\right)\right] / 2},
\end{aligned}
$$

with $\delta_{a b}$ the Kronecker delta and $\mathbf{A}^{\perp}=\mathbf{A}^{\perp}(\mathbf{r}, t)$ and $\mathbf{C}^{\perp}=\mathbf{C}^{\perp}(\mathbf{r}, t)$ the transverse, gauge-invariant pieces of potentials defined generally for the charge-free Maxwell equations such that $\mathbf{E}=-\partial \mathbf{A}^{\perp} / \partial t=-\varepsilon_{0} \mu_{0} \nabla \times \mathbf{C}^{\perp}$ and $\mathbf{B}=\nabla \times \mathbf{A}^{\perp}=-\partial \mathbf{C}^{\perp} / \partial t^{28,29}$ $W, T_{a b}$ and $\mathbf{S}$ can be identified as being (twice the cycle-averaged forms of the electric pieces of) an energy density, ${ }^{15}$ a linear momentum flux density ${ }^{15}$ and a spin density ${ }^{16}$ of the illuminating light whereas $H$ and $N_{a b}$ can be identified as being (the cycleaveraged forms of) a helicity density ${ }^{17,32}$ and an $a b$ infra zilch density ${ }^{18}$ of the same. Whereas the energy and linear momentum of light are rather well established, the helicity, spin and $a b$ infra zilches of light have only recently been scrutinised: they are manifestly intrinsic angular momenta associated with various rotational symmetries inherent in the charge-free Maxwell equations. ${ }^{16-21} X_{a b}$ is unfamiliar to us and vanishes, in fact, for illuminating light comprised of a single plane wave. It is non vanishing in general, however. We could have incorporated $X_{a b}$ into part of $N_{a b}$ whilst retained the interpretation of the latter as being the (cycle-averaged form) of an $a b$ infra zilch density, as $X_{a b}$ is a total divergence which vanishes when integrated over all space. Indeed, the identification of $W, T_{a b}, H, \mathbf{s}, N_{a b}$ and $X_{a b}$ in (8) is neither fundamental nor unique. Nevertheless, it provides us with a simple physical picture.

$\S \beta_{G}{ }^{2}$ and $\beta_{A}{ }^{2}$ can be positive or negative, in spite of the misleading but standard notation. ${ }^{14}$

T For strictly monochromatic light, this helicity density is time independent and cycle averaging is, therefore, inconsequential.

\section{Acknowledgements}

This work was supported by the Carnegie Trust for the Universities of Scotland and the Engineering and Physical Sciences Research Council (EPSRC) grant number EP/I012451/1; "Challenges in Orbital Angular Momentum”. We thank Laurence D Barron for his helpful correspondences and Václav Potoček for his assistance in producing the figures.

\section{References}

1 P. W. Atkins and L. D. Barron, Rayleigh scattering of polarized photons by molecules, Mol. Phys., 1969, 16, 453-466.

2 L. D. Barron and A. D. Buckingham, Rayleigh and Raman scattering from optically active molecules, Mol. Phys., 1971, 20, 1111-1119.

3 D. L. Andrews, Rayleigh and Raman optical activity: An analysis of the dependence on scattering angle, J. Chem. Phys., 1980, 72, 4141-4144.

4 D. P. Craig and T. Thirunamachandran, Molecular Quantum Electrodynamics: An Introduction to Radiation Molecule Interactions, Dover, New York, 1998.

5 L. D. Barron, Molecular Light Scattering and Optical Activity, Cambridge University Press, Cambridge, 2004.

6 L. D. Barron and A. D. Buckingham, Rayleigh and Raman scattering by molecules in magnetic fields, Mol. Phys., 1972, 23, 145-150.

7 A. D. Buckingham and R. E. Raab, Electric-field-induced differential scattering of right and left circularly polarized light, Proc. R. Soc. London, Ser. A, 1975, 345, 365-377.

8 L. D. Barron, M. P. Bogaard and A. D. Buckingham, Raman scattering of circularly polarized light by optically active molecules, J. Am. Chem. Soc., 1973, 95, 603-605.

9 L. D. Barron, Magnetic vibrational optical activity in the resonance Raman spectrum of ferrocytochrome c, Nature, 1975, 257, 372-374.

10 A. D. Buckingham and R. A. Shatwell, Linear electro-optic effect in gases, Phys. Rev. Lett., 1980, 45, 21-25.

11 L. D. Barron, F. Zhu, L. Hecht, G. E. Tranter and N. W. Isaacs, Raman optical activity: An incisive probe of molecular chirality and biomolecular structure, J. Mol. Struct., 2007, 834, 7-16.

12 L. D. Barron and A. D. Buckingham, Vibrational optical activity, Chem. Phys. Lett., 2010, 492, 199-213.

13 Private communication with Laurence D Barron.

14 G. Züber, P. Wipf and D. N. Beratan, Exploring the optical activity tensor by anisotropic Rayleigh optical activity scattering, ChemPhysChem, 2008, 9, 265-271.

15 J. D. Jackson, Classical Electrodynamics, Wiley, New York, 1999. 16 S. M. Barnett, Rotation of electromagnetic fields and the nature of optical angular momentum, J. Mod. Opt., 2010, 57, 1339-1343.

17 S. M. Barnett, R. P. Cameron and A. M. Yao, Duplex symmetry and its relation to the conservation of optical helicity, Phys. Rev. A: At., Mol., Opt. Phys., 2012, 86, 013845.

18 R. P. Cameron, S. M. Barnett and A. M. Yao, Optical helicity, optical spin and related quantities in electromagnetic theory, New J. Phys., 2012, 14, 053050. 
19 S. J. van Enk and G. Nienhuis, Spin and orbital angular momentum of photons, Europhys. Lett., 1994, 25, 497-501.

20 S. J. van Enk and G. Nienhuis, Commutation rules and eigenvalues of spin and orbital angular momentum of radiation fields, J. Mod. Opt., 1994, 41, 963-977.

21 R. P. Cameron and S. M. Barnett, Electric-magnetic symmetry and Noether's theorem, New J. Phys., 2012, 14, 123019.

22 R. P. Cameron, S. M. Barnett and A. M. Yao, Optical helicity of interfering waves, J. Mod. Opt., 2014, 61, 25-31.

23 M. F. Maestre, C. Bustamante, T. L. Hayes, J. A. Subirana and I. Tinoco, Differential scattering of circularly polarized light by the helical sperm head of the octopus Eledone cirrhosa, Nature, 1982, 298, 773-774.

24 Y. Tang and A. E. Cohen, Optical chirality and its interaction with matter, Phys. Rev. Lett., 2010, 104, 163901.

25 Y. Tang and A. E. Cohen, Enhanced enantioselectivity in excitation of chiral molecules by superchiral light, Science, 2011, 332, 333-336.
26 Y. Aharonov, F. Colombo, I. Sabadini and J. Tollaksen, Some mathematical properties of superoscillations, J. Phys. A: Math. Theor., 2011, 44, 365304.

27 J. Dalibard and C. Cohen-Tannoudji, Laser cooling below the Doppler limit by polarization gradients: simple theoretical models, J. Opt. Soc. Am. B, 1989, 6, 2023-2045.

$28 \mathrm{H}$. Bateman, The Mathematical Analysis of Electrical and Optical Wave-Motion on the Basis of Maxwell's Equations, Cambridge University Press, Cambridge, 1915.

29 R. P. Cameron, On the 'second potential' in electrodynamics, J. Opt., 2014, 16, 015708.

30 S. Guy, L. Guy, A. Bensalah-Ledoux, A. Pereira, V. Grenard, O. Cosso and T. Vautey, Pure organic thin films with high isotropic optical activity synthesised by UV pulsed laser deposition, J. Mater. Chem., 2009, 19, 7093-7097.

31 L. Hecht and L. D. Barron, Rayleigh and Raman optical activity from chiral surfaces, Chem. Phys. Lett., 1994, 225, 525-530.

32 D. J. Candlin, Analysis of the new conservation law in electromagnetic theory, II Nuovo Cimento, 1965, 37, 1390-1395. 